\title{
SINGULAR COMPACTIFICATIONS: THE ORDER STRUCTURE
}

\author{
RICHARD E. CHANDLER AND GARY D. FAULKNER
}

\begin{abstract}
This paper investigates the order structure of the collection of singular compactifications of a locally compact Hausdorff space. In particular we will prove several theorems showing that $\beta X$ is the supremum of these simpler compactifications.
\end{abstract}

1. All spaces considered will be locally compact and Hausdorff. Let $K$ be compact and let $f: X \rightarrow K$ be continuous with $f(X)$ dense in $K$. The singular set, $S(f)$, of $f$ consists of those $p \in K$ such that for each neighborhood of $U$ of $p$, $f^{-1}(U)$ does not have compact closure in $X$. If $S(f)=K$, then $f$ is said to be singular. This concept was originally defined by Cain $[2,3,4]$ and Whyburn $[10,11]$. If $f$ is singular, then the singular compactification of $X$ induced by $f$, denoted by $X \cup_{f} S(f)$, may be defined as follows:

On the set $X \cup K$, basic neighborhoods of points in $X$ remain the same as in $X$. Points in $K$ have neighborhoods of the form $V \cup\left\{f^{-1}(V) \backslash F\right\}$, where $V$ is open in $K$ and $F$ is compact in $X$.

This was first defined in [6] as a generalization of the double circumference construction of Alexandroff and Urysohn [1] and has proved to be a very convenient way of constructing compactifications. As a matter of fact, with a few exceptions, the common compactifications are all of this type.

It is known [9] that the singular compactifications form a complete lower semilattice of the lattice of Hausdorff compactifications of a space $X$. Here we will consider further the role these compactifications have in this lattice. For any compactification $\alpha X$, we let $C_{\alpha}$ denote those functions $f$ in $C^{*}(X)$ which have extension $f^{\alpha}$ to $\alpha X$. $S^{\alpha}\left(S^{*}\right)$ will denote those functions of $C_{\alpha}\left(C^{*}\right)$ which are singular. For a space $X, K_{X}$ will denote the collection of compact subsets of $X$.

Lemma 1. For $f \in C_{\alpha}(X)$ we have $f^{\alpha}(\alpha X \backslash X)=S(f)$.

Proof. By [5, Theorem 1.1], we have

$$
S(f)=\bigcap_{F \in K_{X}} \mathrm{Cl}_{\mathbf{R}}(f(X \backslash F)) .
$$

Now if $F \subseteq X$ is compact, then

$$
\mathrm{Cl}_{\alpha X}(X \backslash F) \cap(\alpha X \backslash X)=\alpha X \backslash X,
$$

Received by the editors April 2, 1986.

1980 Mathematics Subject Classification (1985 Revision). Primary 54D35, 54D40, 54C10.

Key words and phrases. Compactification, remainder, singular set, singular mapping.

(C1987 American Mathematical Society $0002-9939 / 87 \$ 1.00+\$ .25$ per page 
so that

$$
f^{\alpha}(\alpha X \backslash X) \subseteq f^{\alpha}\left(\mathrm{Cl}_{\alpha X}(X \backslash F)\right) \subseteq \mathrm{Cl}_{\mathbf{R}} f(X \backslash F) .
$$

It follows that $f^{\alpha}(\alpha X \backslash X) \subseteq S(f)$.

If $f^{\alpha}(\alpha X \backslash X)$ is not dense in $S(f)$, there is a point $p \in S(f)$ and a closed neighborhood $U$ of $p$ with $U \cap f^{\alpha}(\alpha X \backslash X)=\varnothing$. From this it follows that

$$
G=\left(f^{\alpha}\right)^{-1}(U) \subseteq X \subseteq \alpha X,
$$

and since $U$ is closed in $\mathbf{R}, G$ is closed and hence compact. This contradicts the assumption that $p \in S(f)$. Since $f^{\alpha}(\alpha X \backslash X)$ is closed and dense in $S(f)$, we conclude that $f^{\alpha}(\alpha X \backslash X)=S(f)$.

LEMMA 2. If $f \in S^{\alpha}$, then $\alpha X \geqslant X \cup_{f} S(f)$.

Proof. Define $\Pi: \alpha X \rightarrow X \cup_{f} S(f)$ by

$$
\Pi(t)= \begin{cases}t & \text { if } t \in X, \\ f^{\alpha}(t) & \text { if } t \in \alpha X \backslash X .\end{cases}
$$

Let $V=U \cup\left(f^{-1}(U) \backslash K\right)$ be a basic open set in $X \cup_{f} S(f)$. Then

$$
\begin{aligned}
\Pi^{-1}(V) & =\Pi^{-1}(U) \cup \Pi^{-1}\left\{f^{-1}(U) \backslash K\right\} \\
& =\left(f^{\alpha}\right)^{-1}(U) \cup\left\{f^{-1}(U) \backslash K\right\}=\left(f^{\alpha}\right)^{-1}(U) \backslash K
\end{aligned}
$$

which is open in $\alpha X$. From this we conclude that $\Pi$ is continuous. By Lemma $1, \Pi$ is onto. Hence it is a quotient map, showing that $\alpha X \geqslant X \cup_{f} S(f)$.

As was remarked before, in [9] it is shown that any compactification in the lattice of all compactifications which is below a singular compactification is equivalent to a singular compactification. Thus, the singular compactifications form a complete lower lattice. An example is given which shows that the supremum of two singular compactifications need not be equivalent to a singular compactification. It is also easy to see that

$$
\beta \mathbf{N}=\sup \left\{\mathbf{N} \cup S(f) \mid f \in S^{*}(\mathbf{N})\right\} .
$$

In the following theorems we will extend these results.

In all that follows $\Pi_{\alpha \delta}$ will denote the standard quotient map from the larger compactification $\alpha X$ to the smaller compactification $\delta X$.

THEOREM 1. Let $\alpha X$ be a compactification of $X$, and let $\mathscr{G}$ be a subcollection of $S^{\alpha}$. Then

$$
\alpha X=\sup \left\{X \cup_{f} S(f) \mid f \in \mathscr{G}\right\}
$$

if and only if $\mathscr{G}$ separates points in $\alpha X \backslash X$.

Proof. First suppose that $\mathscr{G}$ separates points of $\alpha X \backslash X$. Let

$$
\gamma X=\sup \left\{X \cup_{f} S(f) \mid f \in \mathscr{G}\right\} .
$$

By Lemma 2, $\gamma X \leqslant \alpha X$. Assume that $\gamma X<\alpha X$. If this is true, then there are two distinct points $p, q \in \alpha X \backslash X$, with $\Pi_{\alpha \gamma}(p)=\Pi_{\alpha \gamma}(q)$. Choose $f \in \mathscr{G}$ such that $f^{\alpha}(p) \neq f^{\alpha}(q)$. Let $\delta X=X \cup_{f} S(f)$. Then $\gamma X \geqslant \delta X$ so that there is a projection $\Pi_{\gamma \delta}: \gamma X \rightarrow \delta X$. Since $f$ has extensions to each of $\delta X, \gamma X$, and $\alpha X$, the following 
diagram commutes:

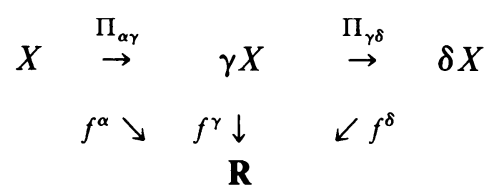

But $f^{\alpha}(p) \neq f^{\alpha}(q)$, while $\Pi_{\alpha \gamma}(p)=\Pi_{\alpha \gamma}(q)$. This is a contradiction so that we conclude that $\alpha X=\gamma X$.

For the converse suppose there are points $p, q \in \alpha X \backslash X$ such that for each $f \in \mathscr{G}$ we have $f^{\alpha}(p)=f^{\alpha}(q)$. Let $\gamma X$ be the compactification formed by identifying $p$ and $q$ in $\alpha X \backslash X$. Since each $f \in \mathscr{G}$ extends to $\gamma X, \gamma X \geqslant X \cup\{(f)$, by Lemma 2. It follows that $\alpha X>\gamma X \geqslant \sup \left\{X \cup_{f} S(f) \mid f \in \mathscr{G}\right\}$.

Lemma 3. Let $X$ be normal, and suppose that $Z_{1}$ and $Z_{2}$ are $C$-embedded in $X$. If $\bar{Z}_{1} \cap \bar{Z}_{2}=\varnothing$, then $Z_{1} \cup Z_{2}$ is $C$-embedded in $X$.

Proof. Let $f \in C\left(Z_{1} \cup Z_{2}\right)$, and let $f_{k}=\left.f\right|_{Z_{k}}$. Choose open sets $U_{1}, V_{1}, U_{2}, V_{2}$ satisfying

(i) $\bar{Z}_{k} \subseteq U_{k} \subseteq \bar{U}_{k} \subseteq V_{k}, k=1,2$,

(ii) $V_{1} \cap V_{2}=\varnothing$.

By Urysohn's lemma there exist continuous functions $g_{k}$ : $X \rightarrow[0,1](k \doteq 1,2)$ such that

(i) $g_{k}\left(\bar{U}_{k}\right)=1$,

(ii) $g_{k}\left(V_{k}^{c}\right)=0$.

Let $\hat{f}_{k}$ denote an extension of $f_{k}$ to $X$. Then $f^{*}=\hat{f}_{1} g_{1}+\hat{f}_{2} g_{2}$ extends $f$ to $X$.

THEOREM 2. Let $X$ be nonpseudocompact. Then $\beta X=\sup \left\{X \cup_{f} S(f) \mid f \in S^{*}\right\}$.

Proof. Let $p, q \in \beta X \backslash X$. Since $X$ is nonpseudocompact it must contain a $C$-embedded copy of the natural numbers. Denote this set by $N_{1}$. Since $\mathrm{Cl}_{\beta X}\left(N_{1}\right)=$ $\beta N_{1}$, there is an $r \in \mathrm{Cl}_{\beta X}\left(N_{1}\right) \cap(\beta X \backslash X)$ with $r \neq p, q$. Let $U$ be a neighborhood of $r$ with $p, q \notin \mathrm{Cl}_{\beta X}(U)$. Let $N=U \cap N_{1} . N$ is a copy of the natural numbers which is $C$-embedded in $X$, and hence in $\beta X$. By Lemma 3, $N \cup\{p, q\}$ is $C$-embedded in $\beta X$. Define a function $f^{*}: N \cup\{p, q\} \rightarrow \mathbf{R}$ by $f^{*}(p)=1, f^{*}(q)=$ 0 , and $f^{*}: N \rightarrow \mathbf{Q} \cap[0,1]$ defined in any way so as to be onto. Let $\hat{f}$ be the extension of $f^{*}$ to $\beta X$, and $f$ the restriction of $\hat{f}$ to $X$. Without loss of generality, we can assume that the range of $f$ is contained in $[0,1]$. Now $f \in S^{*}$ and $f^{\beta}(p) \neq f^{\beta}(q)$. Thus $S^{*}$ separates points in $\beta X \backslash X$, which by Theorem 1, concludes the proof.

Corollary to the Proof of Theorem 2. Let $X$ be nonpseudocompact. Then $\beta X$ is the supremum of compactifications having connected remainders. In particular these remainders may each be taken to be homeomorphic to the unit interval $[0,1]$.

Proof. The function $f$ constructed in the proof of Theorem 2 has as singular set the entire interval $[0,1]$. Hence we may assume that the separating family of functions is of this form. The result follows from this. 
We might point out that this result seems to set $\beta X$ in contradistinction to the Freudenthal compactification, which is the supremum of compactifications with totally disconnected remainders. It is of course possible, this theorem nonwithstanding, that $\beta X$ and the Freudenthal compactification coincide. In particular $\beta \mathbf{N}$ is, either by Theorem 1 or elementary observation, the supremum of singular compactifications having two point remainders. $\mathscr{G}$ is the collection of all functions which are obtained by partitioning $\mathbf{N}$ into two infinite disjoint subsets and mapping one of these onto 0 an the other onto 1 .

Recall that a space $X$ is said to be 1-complemented (or connected at infinity) provided each compact set $K$ is contained in a compact set $F$ with $X \backslash F$ connected.

TheOREM 3. If $X$ is 1-complemented, then $\beta X=\sup \left\{X \cup_{f} S(f) \mid f \in S^{*}\right\}$.

Proof. Let $p, q \in \beta X \backslash X$, with $p \neq q$. Let $f \in C(\beta X)$ be chosen such that $f(p)=0, f(q)=1$, and the range of $f$ is contained in [0,1]. Since $\beta X \backslash X$ is connected, by Lemma 1 , the restriction of $f$ to $X$ has $[0,1]$ as its singular set. Thus the functions in $S^{*}$ separate the points of $\beta X \backslash X$.

2. A topological space $X$ is said to be retractive provided there is a retraction from $\beta X$ onto the remainder $\beta X \backslash X$. Retractive spaces were introduced by $\mathrm{W}$. W. Comfort [7] who proved that a retractive space must be both locally compact and pseudocompact. Comfort's proof of this theorem rested on the Continuum Hypothesis. Subsequently this dependence was removed by van Douwen [12] and Conway [8]. In [9] it is shown that a space is retractive if and only if $\beta X$ is singular.

If for some space $X, \beta X$ fails to be the supremum of singular compactifications in the sense of Theorem 2, then $X$ must of course be pseudocompact. The following theorems show that pseudocompact spaces may have this property as well.

THEOREM 4. Let $X$ be retractive. Then each $f \in C^{*}(X)$ can be written as $f=f_{s}+f_{\infty}$, where $f_{s} \in S^{*}(X)$ and $f_{\infty} \in C_{\infty}(X)$.

Proof. Let $r: \beta X \rightarrow \beta X \backslash X$ be the retraction. From the proof of Comfort's theorem in [8], there is a bounded linear projection $P: C^{*}(X) \rightarrow C_{\infty}(X)$, where $(I-P) f=\left.f \circ r\right|_{X}$ for each $f \in C^{*}(X)$. Clearly $f_{s}=\left.f \circ r\right|_{X}$ is singular since the composition of a singular function with a continuous function is singular. Let $f_{\infty}=P f ;$ then

$$
f=P f+(I-P) f=f_{\infty}+f_{s} .
$$

TheOREM 5. Let $X$ be retractive. Then $\beta X=\sup \left\{X \cup_{f} S(f) \mid f \in S^{*}\right\}$.

Proof. Let $\gamma X=\sup \left\{X \cup_{f} S(f) \mid f \in S^{*}\right\}$. It will suffice to show that each $f \in C^{*}(X)$ has an extension to $\gamma X$. Let $f \in C^{*}(X)$. Let $f_{s}, f_{\infty}$ be as in Theorem 3 . Now $f_{s}$ extends to $X \cup_{f_{s}} S\left(f_{s}\right)$ and hence to $\gamma X$. But $f_{\infty}$ extends to every compactification of $X$, so that $f$ extends to $\gamma X$. It follows that $\gamma X=\beta X$.

THEOREM 6. If the set of singular compactifications forms a lattice, then it forms a complete lattice.

Proof. By [9] the singular compactifications form a complete lower lattice. Suppose $S K(X)$, the set of singular compactifications of $X$, is an upper lattice and 
suppose

$$
\mathscr{T}=\left\{\alpha_{\iota} X \mid \alpha_{\iota} X=X \cup_{f_{\iota}} S\left(f_{\iota}\right), \iota \in I\right\}
$$

is a subset of $S K(X)$. By assumption, the supremum of any finite subcollection of $\mathscr{F} \subseteq \mathscr{T}$ is singular. This supremum is realized as the singular compactification generated by the mapping

$$
h_{\mathscr{F}}: X \rightarrow \prod_{\alpha_{\iota} \in \mathscr{F}} S\left(f_{\imath}\right)
$$

defined by $\left\langle h_{\mathscr{F}}(x)\right\rangle_{\iota}=f_{\iota}(x), \iota \in I$ [6]. Let $h: X \rightarrow \Pi_{\iota \in I} S\left(f_{\iota}\right)$ be defined similarly: $\langle h(x)\rangle_{\iota}=f_{\iota}(x), \iota \in I$. If $h$ is not singular then there exist a basic open set $U=\prod_{\iota \in I} U_{\iota}$ in $\Pi_{\iota \in I} S\left(f_{\iota}\right)$ with $\mathrm{Cl}_{X}\left(h^{-1}(U)\right)$ compact. But $h^{-1}(U)=\bigcap_{k=1}^{n} f^{-1}\left(U_{\iota}\right)$, where $\left\{U_{\iota_{k}}\right\}$ denotes the nontrivial factors of $U$, so that the mapping $h_{\mathscr{F}}$ with $\mathscr{F}=\left\{\alpha_{\iota_{k}} X \mid k=1, \ldots, n\right\}$ is not singular.

COROllary. If $X$ is nonpseudocompact, then there exist singular compactifications $\alpha_{1} X$ and $\alpha_{2} X$ for which $\sup \left\{\alpha_{1} X, \alpha_{2} X\right\}$ is not singular.

Proof. By Theorem 6, if $\sup \left\{\alpha_{1} X, \alpha_{2} X\right)$ is singular for each pair of singular compactifications $\alpha_{1} X, \alpha_{2} X$, then the singular compactifications form a complete lattice. But Theorem 2 would then imply that $\beta X$ is singular and hence $X$ would be retractive [9]. By Comfort's Theorem [7] a retractive space must be pseudocompact. This contradiction completes the proof.

THEOREM 7. $X$ is retractive if and only if $S^{*}$.

(i) Each $f$ in $C^{*}(X)$ can be written as $f=f_{\infty}+f_{s}$, where $f_{\infty}$ is in $C_{\infty}$ and $f_{s}$ is in

(ii) The singular compactifications of $X$ form a lattice.

Proof. Suppose that $X$ is retractive. Then Theorem 4 implies (i) and (ii) follows from the fact that $\beta X$ itself is a singular compactification and hence the entire lattice consists of singular compactifications. To reverse the implication we note as in Theorem 5 that (i) implies that $\beta X$ is the supremum of singular compactifications. Thus (ii) together with Theorem 6 implies that $\beta X$ is singular. As remarked previously, this is equivalent to $X$ being retractive.

\section{REFERENCES}

1. P. S. Alexandroff and P. Urysohn, Memoire sur les espaces topologiques compacts, Verh. Nederl. Akad. Wetensch. Afd. Natuurk. Sect. I 14 (1929), 1-96.

2. George L. Cain, Jr., Compact and related mappings, Duke Math. J. 33 (1966), 639-645.

3. ___ Mappings with prescribed singular sets, Nieuw Arch. Wisk. (3) 17 (1969), 200-203.

4. (1980), 311-315.

5. George L. Cain, Jr., Richard E. Chandler, and Gary D. Faulkner, Singular sets and remainders, Trans. Amer. Math. Soc. 268 (1981), 161-171.

6. Richard E. Chandler, Gary D. Faulkner, Josephine P. Guglielmi, and Margaret Memory, Generalizing the Alexandroff-Urysohn double circumference construction, Proc. Amer. Math. Soc. 83 (1981), 606-608. 
7. W. W. Comfort, Retractions and other continuous maps from $\beta X$ to $\beta X \backslash X$, Trans. Amer. Math. Soc. 114 (I1965), 1-9.

8. John B. Conway, Projections and retractions, Proc. Amer. Math. Soc. 1 (1966), 843-847.

9. Josephine P. Guglielmi, Compactifications with singular remainders, $\mathrm{Ph}$. D. Thesis, North Carolina State University, 1986.

10. G. T. Whyburn, Compactifications of mappings, Math. Ann. 166 (1966), 168-174.

11. Dynamic topology, Amer. Math. Monthly 77 (1970), 556-570.

12. Eric K. van Douwen, Retractions from $\beta X$ onto $\beta X \backslash X$, General Topology and Appl. 9 (1978), 169-173.

Department of Mathematics, North Carolina State University, Raleigh, North Carolina 27695-8205 